\title{
EXISTENCE OF EXCEPTIONAL POINTS FOR FUCHSIAN GROUPS OF FINITE COAREA
}

\author{
TOSHIHIRO NAKANISHI AND AKIRA USHIJIMA
}

Abstract. It is shown by Fera that there exists uncountably many exceptional points for cocompact Fuchsian groups. We generalize this result to the case that Fuchsian groups are of finite coarea.

\section{INTRODUCTION}

Let $G$ be a Fuchsian group of finite coarea acting on the hyperbolic plane $\mathbb{H}^{2}$. Such a group must be finitely generated, and every Dirichlet polygon has finitely many sides (see, for example, Theorem 10.1.2 in [2]). A point $z \in \mathbb{H}^{2}$ that is not fixed by any non-trivial element of $G$ is said to be regular if the number of the sides of the Dirichlet polygon $D(z)$ centered at $z$ is maximal, and exceptional otherwise. If $G$ is of type $(g, m)$, then the number of the sides of the Dirichlet polygon $D(z)$ with center $z \in \mathbb{H}^{2}$ is at most $12 g-4 m-6$. So a point $z$ is regular if and only if the number of the sides of $D(z)$ is $12 g-4 m-6$. It is shown by Theorem 9.4.5 in 2] that generic points are regular.

Joseph Fera studied in 3] the existence of exceptional points for cocompact Fuchsian groups; it is shown that the set of exceptional points is uncountable. We generalize this result to Fuchsian groups of finite coarea. The following is our main result, which will be proved as Theorem 5.1

Theorem. For any Fuchsian group of finite coarea, the set of exceptional points contains uncountably many points.

The statement of this theorem is the same as in the case for cocompact Fuchsian groups. However, several phenomena which are not observed for cocompact Fuchsian groups make Fera's proof as it is not enough to obtain the result. For example, if $G$ is of finite coarea but not cocompact, then, as Umemoto's result shows (see Example 5.2), there exists a case where the Dirichlet polygons $D\left(z_{n}\right)$ for a converging sequence $z_{n}$ to $z$ have a common side-pairing transformation $g$ and the sides $s_{n}$ and $g\left(s_{n}\right)$ of $D\left(z_{n}\right)$ paired by $g$ eventually leave any compact set including $z$. It is also possible that the set of points having the same collection of side-pairing transformations is unbounded, contrary to the case of cocompact Fuchsian groups. These facts forced us to employ several additional arguments as presented in the whole sections.

\section{Preliminaries}

2.1. Dirichlet polygons. Let $\mathbb{H}^{2}$ be the hyperbolic plane with distance function $\rho$. The circle at infinity is denoted by $\partial_{\infty} \mathbb{H}^{2}$. For a subset $A \subset \mathbb{H}^{2}$, we denote its

Received by the editors September 30, 2019, and, in revised form, June 22, 2020.

2010 Mathematics Subject Classification. Primary $20 \mathrm{H} 10$. 
closure and boundary relative to $\mathbb{H}^{2}$ by $\bar{A}$ and $\partial A$, respectively. The open disk in $\mathbb{H}^{2}$ with radius $r>0$ and center $z \in \mathbb{H}^{2}$ is denoted by $B_{r}(z):=\left\{w \in \mathbb{H}^{2} \mid \rho(z, w)<r\right\}$.

Let $G$ be a Fuchsian group, that is, a discrete group of orientation-preserving isometries of $\mathbb{H}^{2}$. For such a group $G$ and a point $z \in \mathbb{H}^{2}$, we introduce a polygon, called the Dirichlet polygon, $D(z)$ following [2]. Let $\operatorname{Fix}(G)$ be the set of points in $\mathbb{H}^{2}$ that are fixed by a non-trivial element of $G$ :

$$
\operatorname{Fix}(G):=\left\{z \in \mathbb{H}^{2} \mid g(z)=z \text { for some } g \in G-\{\operatorname{id}\}\right\},
$$

where id represents the trivial element of $G$, which is also the identity mapping on $\mathbb{H}^{2}$. For an isometry $f$ on $\mathbb{H}^{2}$, we denote by $H_{f}(x)$ the set of points in $\mathbb{H}^{2}$ which are closer to $z$ than $f(z)$, and its boundary by $L_{f}(z)$ :

$$
\begin{aligned}
H_{f}(z) & :=\left\{w \in \mathbb{H}^{2} \mid \rho(w, z)<\rho(w, f(z))\right\} \\
L_{f}(z) & :=\partial H_{f}(z)=\left\{w \in \mathbb{H}^{2} \mid \rho(w, z)=\rho(w, f(z))\right\} .
\end{aligned}
$$

For $z \in \mathbb{H}^{2}-\operatorname{Fix}(G)$, let $D(z)$ be the Dirichlet polygon for $G$ with center $z$ :

$$
D(z):=\bigcap_{g \in G-\{\operatorname{id}\}} H_{g}(z)
$$

It is known that Dirichlet polygons are convex and locally finite fundamental domains for $G$; see Theorem 9.4.2 in [2].

A vertex of $D(z)$ is a single point of the form $\overline{D(z)} \cap g(\overline{D(z)}) \cap h(\overline{D(z)})$ for some distinct elements $g, h \in G-\{\mathrm{id}\}$. A side of $D(z)$ is a geodesic segment of the form $\overline{D(z)} \cap g(\overline{D(z)})$ of positive length for some $g \in G-\{$ id $\}$. So, for each side $s$, there exists a unique element $g \in G$ such that $g(s)$ is another side of $D(z)$. Such an element is called a side-pairing transformation of $D(z)$, and we denote the set of all side-pairing transformations of $D(z)$ by $S(z)$. The set $S(z)$ not only generates $G$, but its elements also have the following property (see Chapter 9 in 2 for the proof).

Proposition 2.1. Let $G$ be a Fuchsian group and suppose that $g \in G$.

(1) Let $s$ be a side of $D(z)$. The side $s$ is given by $\overline{D(z)} \cap g(\overline{D(z)})$ if and only if $s \subset L_{g}(z)$.

(2) The element $g$ is in $S(z)$ if and only if $D(z)$ has a side given by $\overline{D(z)} \cap$ $g^{-1}(\overline{D(z)})$.

(3) If $g \in S(z)$, then $g^{-1} \in S(z)$.

The terminology of vertices are generalized to the points on $\partial_{\infty} \mathbb{H}^{2}$. Let $E(z)$ be the set of points on the boundary of $D(z)$ relative to $\mathbb{H}^{2} \cup \partial_{\infty} \mathbb{H}^{2}$, but not on the boundary $\partial D(z)$ of $D(z)$ relative to $\mathbb{H}^{2}$. A free side of $D(z)$ is a maximal interval in $E(z)$. A point $v \in E(z)$ is called a proper vertex if it is the endpoint of two sides of $D(z)$, and called an improper vertex if it is the endpoint of a side and a free side of $D(z)$.

We introduce two types of characterizations of Dirichlet polygons with respect to their combinatorial structure. The first one is a generic Dirichlet polygon. For a point $v$ on the boundary of $D(z)$ relative to $\mathbb{H}^{2} \cup \partial_{\infty} \mathbb{H}^{2}$, its cycle is the intersection of $G(v)$ with this boundary, where $G(v)$ is the $G$-orbit of $v$ :

$$
G(v):=\left\{w \in \mathbb{H}^{2} \cup \partial_{\infty} \mathbb{H}^{2} \mid w=g(v) \text { for some } g \in G\right\} .
$$


The number of points in the cycle is called its length. A vertex, a proper vertex, or an improper vertex is said to be accidental if the length of its cycle is greater than 2 .

Definition 2.2. For a Fuchsian group $G$ and for $z \in \mathbb{H}^{2}-\operatorname{Fix}(G)$, the Dirichlet polygon $D(z)$ is said to be generic if it satisfies the following conditions:

(1) The cycle of a vertex of $D(z)$ fixed by an elliptic element of $G$ has length 1.

(2) The cycle of an accidental vertex of $D(z)$ in $\mathbb{H}^{2}$ has length 3.

(3) The cycle of an improper vertex of $D(z)$ that is not a limit point of $G$ has length 2 .

(4) The cycle of a proper vertex of $D(z)$ has length 1 , and the cycle consists of a parabolic fixed point.

(5) The cycle of a parabolic fixed point on the boundary of $D(z)$ relative to $\mathbb{H}^{2} \cup \partial_{\infty} \mathbb{H}^{2}$ has length 1 , and the cycle consists of a proper vertex.

A point in $\mathbb{H}^{2}$ is said to be generic if it is the center of a generic Dirichlet polygon.

We remark that almost all points in $\mathbb{H}^{2}$ are generic by Theorem 9.4.5 in [2]

Before introducing the other characterization of Dirichlet polygons, we introduce the type of a Fuchsian group. For any $z \in \mathbb{H}^{2}$, the orbit $G(z)$ has no accumulation points in $\mathbb{H}^{2}$ as $G$ acts discontinuously on $\mathbb{H}^{2}$. The resulting quotient surface $\mathbb{H}^{2} / G$ becomes a hyperbolic orbifold with complete hyperbolic metric induced from $\rho$. We denote the canonical projection from $\mathbb{H}^{2}$ to $\mathbb{H}^{2} / G$ by $\pi$. A Fuchsian group $G$ is said to be of finite coarea if the area of $\mathbb{H}^{2} / G$ is finite. We mainly consider these groups in what follows. Let $m$ be the number of conjugacy classes of maximal parabolic or maximal elliptic cyclic subgroups of $G$. We remark that $m$ is a finite number, and that each maximal parabolic cyclic subgroup corresponds to a puncture on $\mathbb{H}^{2} / G$. Let $g$ be the genus of $\mathbb{H}^{2} / G$. Then the group $G$ is said to be of type $(g, m)$. The maximal number of sides of Dirichlet polygons for a Fuchsian group of finite coarea is characterized by its type in the following proposition.

Proposition 2.3. For a Fuchsian group $G$ of finite coarea, if it is of type $(g, m)$, then the maximal number $S_{G}$ of sides is $12 g+4 m-6$. Furthermore, a Dirichlet polygon has $S_{G}$ sides if and only if it is generic.

Proof. By the proof of Theorem 10.5.1 of [2], the Dirichlet polygon $D(z)$ has $S_{G}=$ $12 g+4 m-6$ sides if and only if all cycles of parabolic and elliptic fixed points on the vertices of $D(z)$ have length 1 and all accidental cycles have length 3 .

Definition 2.4. Let $G$ be a Fuchsina group of finite coarea. The Dirichlet polygon $D(z)$ is said to be regular if it has $S_{G}$ sides. A point $z \in \mathbb{H}^{2}-\operatorname{Fix}(G)$ is said to be regular if it is the center of a regular Dirichlet polygon, and exceptional otherwise.

We denote by $\operatorname{Reg}(G)$ and $\operatorname{Exc}(G)$ the set of regular points and the set of exceptional points, respectively. We remark that these sets together with $\operatorname{Fix}(G)$ disjointly decompose $\mathbb{H}^{2}$. Theorem 9.4.5 in 2 together with Proposition 2.3 implies that almost all points in $\mathbb{H}^{2}$ are regular. This conclusion, however, does not mean that $\operatorname{Exc}(G)$ is non-empty. Our main result, Theorem 5.1, is that $\operatorname{Exc}(G)$ is actually non-empty for every Fuchsian group $G$ of finite coarea.

We close this subsection with a characterization of proper vertices for Fuchsian groups of finite coarea. We first remark that every point in $E(z)$ is a proper vertex when $G$ is of finite coarea. Furthermore, we have the following result. 
Lemma 2.5. Each proper vertex of a Fuchsian group of finite coarea is a parabolic fixed point.

Proof. For a Fuchsian group $G$ of finite coarea, let $p$ be a proper vertex of $D(z)$ that is an endpoint of a side $s$. Suppose that a point $\zeta$ on $s$ approaches $p$. Then the distance $\rho(z, \zeta)$ diverges. By the definition of the Dirichlet polygon $D(z)$, the distance $\rho(z, \zeta)$ coincides with the distance from $\pi(z)$ to $\pi(\zeta)$ on the quotient surface $\mathbb{H}^{2} / G$. Hence $\pi(\zeta)$ tends to a puncture of $\mathbb{H}^{2} / G$.

2.2. Truncated Dirichlet polygons. If a Fuchsian group $G$ is of finite coarea but not cocompact, then its Dirichlet polygon cannot be compact. To proceed with our argument, we need to prepare suitable compact subsets of the Dirichlet polygons under consideration. Such subsets will be called truncated Dirichlet polygons.

To define truncated Dirichlet polygons, we use a particular model of $\mathbb{H}^{2}$; from here until the proof of Theorem 5.1, we regard $\mathbb{H}^{2}$ as the so-called upper half-plane model, i.e.,

$$
\mathbb{H}^{2}:=\{z=x+i y \in \mathbb{C} \mid x, y \in \mathbb{R} \text { and } y>0\}
$$

equipped with $d s^{2}=\left(d x^{2}+d y^{2}\right) / y^{2}$, where $i$ means the imaginary unit. The orientation-preserving isometry group is then isomorphic to the special linear group $\mathrm{SL}(2, \mathbb{R})$ of degree 2 over $\mathbb{R}$, and the circle at infinity $\partial_{\infty} \mathbb{H}^{2}$ is then described as $\mathbb{R} \cup\{\infty\}$, where $\infty$ means the point at infinity. The hyperbolic distance $\rho(z, w)$ for $z, w \in \mathbb{H}^{2}$ is given by

$$
\sinh \frac{\rho(z, w)}{2}=\frac{|z-w|}{2 \sqrt{\operatorname{Im}[z]} \sqrt{\operatorname{Im}[w]}} .
$$

See Theorem 7.2.1 in [2].

Before introducing the truncated Dirichlet polygon, we review properties of parabolic elements in a Fuchsian group. Let $G$ be a Fuchsian group containing parabolic elements, and let $p \in \partial_{\infty} \mathbb{H}^{2}$ be a parabolic fixed point of $G$. A parabolic element $f \in G$ is said to be primitive if it generates the stabilizer

$$
G_{p}:=\{g \in G \mid g(p)=p\}
$$

of $p$. For such a parabolic primitive element $f$ with respect to $G_{p}$ and for any $g \in G, g f g^{-1}$ is a primitive element with respect to $G_{g(p)}$.

For each parabolic fixed point of a Fuchsian group $G$, we introduce two kinds of sets in $\mathbb{H}^{2}$, called a horodisk and a horocycle. We first consider the case that $\infty$ is a parabolic fixed point of $G$ and that

$$
t:=\left(\begin{array}{ll}
1 & 1 \\
0 & 1
\end{array}\right) \in \mathrm{SL}(2, \mathbb{R})
$$

is a primitive element of $G_{\infty}$; otherwise take a conjugation of $G$ to $h G h^{-1}$ for a suitable element $h \in \mathrm{SL}(2, \mathbb{R})$ with $h(p)=\infty$ for a parabolic fixed point $p$. For any $r \in \mathbb{R}$, the horodisk $\mathscr{D}_{r}(\infty)$ and the horocycle $\mathscr{H}_{r}(\infty)$ at $\infty$ are defined as

$$
\begin{aligned}
& \mathscr{D}_{r}(\infty):=\left\{z \in \mathbb{H}^{2} \mid \operatorname{Im}[z]>e^{r}\right\}, \\
& \mathscr{H}_{r}(\infty):=\partial \mathscr{D}_{r}(\infty)=\left\{z \in \mathbb{H}^{2} \mid \operatorname{Im}[z]=e^{r}\right\} .
\end{aligned}
$$

Let $p \in \mathbb{R}=\partial_{\infty} \mathbb{H}^{2}-\{\infty\}$ be a parabolic fixed point which is $G$-equivalent to $\infty$, i.e., there exists some $h \in G$ such that $p=h(\infty)$. This $h$ can be taken such a way that $h^{-1} f h=t$ for some primitive element $f$ of $G_{p}$. The horodisk $\mathscr{D}_{r}(p)$ and the 
horocycle $\mathscr{H}_{r}(p)$ at $p$ are then defined as $h\left(\mathscr{D}_{r}(\infty)\right)$ and $h\left(\mathscr{H}_{r}(\infty)\right)$, respectively. We remark that both $h\left(\mathscr{D}_{r}(\infty)\right)$ and $h\left(\mathscr{H}_{r}(\infty)\right)$ are independent of the choice of $h$.

The following lemma, which is a corollary of the Shimizu-Leutbecher inequality, is well known. See Corollary 5.3 in [1].

Lemma 2.6. Let $G$ be a Fuchsian group, and let $p$ be a parabolic fixed point of $G$. Then, for any $r \in \mathbb{R}$ and any $g \in G, g\left(\mathscr{D}_{r}(p)\right)=\mathscr{D}_{r}(g(p))$ holds. Furthermore, for any $r>0$ and any distinct but $G$-equivalent parabolic fixed points $p$ and $q$, $\overline{\mathscr{D}_{r}(p)} \cap \overline{\mathscr{D}_{r}(q)}=\emptyset$ holds.

An immediate consequence of Lemma 2.6 is that for any $r>0$, the closure of a horodisk $\overline{\mathscr{D}_{r}(p)}$ is precisely invariant under $G_{p}$, that is, $g^{m}\left(\overline{\mathscr{D}_{r}(p)}\right)=\overline{\mathscr{D}_{r}(p)}$ holds for a primitive element $g \in G_{p}$ and any integer $m$, and $\overline{\mathscr{D}_{r}(p)} \cap h\left(\overline{\mathscr{D}_{r}(p)}\right)=\emptyset$ holds for any $h \in G-G_{p}$.

Proposition 2.7. Let $G$ be a Fuchsian group of finite coarea but not cocompact. Then there exists some $R \in \mathbb{R}$ such that for any $r \geq R$ and any distinct parabolic fixed points $p$ and $q$ of $G, \overline{\mathscr{D}_{r}(p)} \cap \overline{\mathscr{D}_{r}(q)}=\emptyset$ holds.

Proof. For any $r>0$ and any $G$-equivalent distinct parabolic fixed points $p$ and $q$, $\overline{\mathscr{D}_{r}(p)} \cap \overline{\mathscr{D}_{r}(q)}=\emptyset$ holds by Lemma 2.6. There exist only finitely many $G$-equivalent classes of parabolic fixed points as $G$ is of finite coarea. We can thus take $R$ to be a sufficiently large positive number, and for any $r \geq R$ and any distinct parabolic fixed points $p$ and $q$, which are not necessarily $G$-equivalent, $\overline{\mathscr{D}_{r}(p)} \cap \overline{\mathscr{D}_{r}(q)}=\emptyset$ holds.

For $R \in \mathbb{R}$ chosen from Proposition 2.7 and for any $r \geq R$, we define the truncated Nielsen region $N_{r}$ as

$$
N_{r}:=\mathbb{H}^{2}-\bigcup_{p} \overline{\mathscr{D}_{r}(p)},
$$

where $p$ runs over all parabolic fixed points of $G$. Since $\pi\left(\bigcup_{p} \overline{\mathscr{D}_{r}(p)}\right)$ is a finite disjoint union of closed cusp neighborhoods on $\mathbb{H}^{2} / G$, the set $\pi\left(N_{r}\right)$ is a bounded open set that is isotopic to the hyperbolic orbifold $\mathbb{H}^{2} / G$. Let $M_{r}$ be the diameter of the compact subset $\pi\left(\overline{N_{r}}\right)$ in $\mathbb{H}^{2} / G$.

For each point $z \in \mathbb{H}^{2}-\operatorname{Fix}(G)$, we define the truncated Dirichlet polygon, by

$$
D_{r}(z):=D(z) \cap N_{r}=D(z)-\bigcup_{p} \overline{\mathscr{D}_{r}(p)} .
$$

This (open) polygon is bounded in $\mathbb{H}^{2}$. By the definition of the Dirichlet polygon, for any point $w \in D(z)$, the distance $\rho(z, w)$ is the shortest among the distances $\rho(z, g(w))$ for any $g \in G$. Therefore, for any $z \in \mathbb{H}^{2}-\operatorname{Fix}(G)$, the diameter of $D_{r}(z)$ is bounded above by $2 M_{r}$.

The boundary $\partial D_{r}(z)$ of $D_{r}(z)$ consists of two types of points; whether it comes from the boundary of $D(z)$ or not. The set of points of the former type is denoted by $\partial_{D} D_{r}(z)$ :

$$
\partial_{D} D_{r}(z):=\partial D_{r}(z) \cap \partial D(z)=\partial D(z)-\bigcup_{p} \overline{\mathscr{D}_{r}(p)} .
$$

The truncated Dirichlet polygon $D_{r}(z)$ is a "Dirichlet polygon" of $G$ with respect to $N_{r}$ in the following sense: we first remark that $\pi\left(N_{r}\right)=\pi\left(D_{r}(z)\right)$ by the 
definition of $D_{r}(z)$. We mean a side of $D_{r}(z)$ as the restriction of a side of $D(z)$ on $\partial_{D} D_{r}(z)$.

If a Fuchsian group $G$ is of finite coarea but not cocompact, then the center $z$ of $D(z)$ might not lie in $D_{r}(z)$, for $z$ could lie in $\overline{\mathscr{D}_{r}(p)}$. This is a significant difference between cocompact Fuchsian groups and Fuchsian groups of finite coarea. The following lemma characterizes the shape of $D(z)$ when $z$ is sufficiently close to a parabolic fixed point.

Lemma 2.8. Let $G$ be a Fuchsian group, and let $p$ be a parabolic fixed point of $G$. Then, for any $z \in \mathscr{D}_{1 / 2}(p)-\operatorname{Fix}(G)$, there exists some $R \in \mathbb{R}$ such that for any $r \geq R, \partial D(z) \cap \mathscr{D}_{r}(p)$ consists of two sides that are paired by a primitive element of $G_{p}$.

Proof. We assume without loss of generality that the parabolic fixed point $p$ in question is $\infty$ and that $t$ of $(2.2)$ is primitive with respect to $G_{\infty}$. By conjugating $G$ with a translation $z \mapsto z+\ell$ with a suitable real number $\ell$, we additionally assume that the center $z$ of a Dirichlet polygon is represented as $i e^{\zeta}$ for some $\zeta \in \mathbb{R}$. Then we have $\zeta>1 / 2$ by $z=i e^{\zeta} \in \mathscr{D}_{1 / 2}(\infty)$.

For $R \in \mathbb{R}$, let

$$
\begin{aligned}
\mathscr{S}_{R} & :=H_{t}\left(i e^{\zeta}\right) \cap H_{t^{-1}}\left(i e^{\zeta}\right) \cap \mathscr{D}_{R}(\infty) \\
& =\left\{w \in \mathbb{H}^{2} \mid-1 / 2<\operatorname{Re}[w]<1 / 2, \operatorname{Im}[w]>e^{R}\right\} .
\end{aligned}
$$

Our aim is to find some $R \in \mathbb{R}$ which satisfies $D\left(i e^{\zeta}\right) \cap \mathscr{D}_{R}(\infty)=\mathscr{S}_{R}$. Then, for any $r \geq R$, we also have $D\left(i e^{\zeta}\right) \cap \mathscr{D}_{r}(\infty)=\mathscr{S}_{r}$, since $\mathscr{D}_{r}(\infty) \subset \mathscr{D}_{R}(\infty)$.

For any $R \in \mathbb{R}, D\left(i e^{\zeta}\right) \cap \mathscr{D}_{R}(\infty) \subset \mathscr{S}_{R}$ holds by the definition of the Dirichlet polygon. So we need to find $R \in \mathbb{R}$ which satisfies $D\left(i e^{\zeta}\right) \cap \mathscr{D}_{R}(\infty) \supset \mathscr{S}_{R}$, or equivalently $\rho\left(w, i e^{\zeta}\right)<\rho\left(w, g\left(i e^{\zeta}\right)\right)$ for any $w \in \mathscr{S}_{R}$ and any $g \in G-\{$ id $\}$.

We first suppose that $g \in G_{\infty}-\{\mathrm{id}\}$. Then there exists some $m \in \mathbb{N}$ such that $g=t^{m}$. So $g\left(i e^{\zeta}\right)=m+i e^{\zeta}$. A straightforward calculation by (2.1) shows that for any $w \in H_{t}\left(i e^{\zeta}\right) \cap H_{t^{-1}}\left(i e^{\zeta}\right), \rho\left(w, i e^{\zeta}\right)<\rho\left(w, m+i e^{\zeta}\right)$ holds.

We next suppose that $g \in G-G_{\infty}$, and we will see that $R$ can be taken as $\zeta$; let $R:=\zeta$ from now on. We first show that for any $w \in \mathscr{S}_{\zeta}, \rho\left(w, g\left(i e^{\zeta}\right)\right) \geq$ $\rho\left(w, \mathscr{H}_{-\zeta}(\infty)\right)$ holds. The point $g\left(i e^{\zeta}\right)$ is on the horocycle $g\left(\mathscr{H}_{\zeta}(\infty)\right)$ as $i e^{\zeta} \in$ $\mathscr{H}_{\zeta}(\infty)$. This horocycle is a Euclidean circle with diameter $|c|^{-2} e^{-\zeta}$ being tangent to the real axis if

$$
g=\left(\begin{array}{ll}
a & b \\
c & d
\end{array}\right) .
$$

Hence $\operatorname{Im}\left[g\left(i e^{\zeta}\right)\right] \leq e^{-\zeta}$ by the Shimizu-Leutbecher inequality. The hyperbolic segment between $w$ and $g\left(i e^{\zeta}\right)$ meets $\mathscr{H}_{0}(\infty)=\left\{w \in \mathbb{H}^{2} \mid \operatorname{Im}[w]=1\right\}$ at a point, say $w_{0}$, since $\operatorname{Im}[w]>e^{\zeta}$ and $r>0$. Then we have

$$
\begin{aligned}
\rho\left(w, g\left(i e^{\zeta}\right)\right) & =\rho\left(w, w_{0}\right)+\rho\left(w_{0}, g\left(i e^{\zeta}\right)\right) \\
& \geq \rho\left(w, \mathscr{H}_{0}(\infty)\right)+\rho\left(w_{0}, \mathscr{H}_{-\zeta}(\infty)\right) \\
& \geq \rho\left(w, \mathscr{H}_{0}(\infty)\right)+\rho\left(\mathscr{H}_{0}(\infty), \mathscr{H}_{-\zeta}(\infty)\right) \\
& =\rho\left(w, \mathscr{H}_{-\zeta}(\infty)\right) .
\end{aligned}
$$

We next show that for any $w \in \mathscr{S}_{\zeta}, \rho\left(w, \mathscr{H}_{-\zeta}(\infty)\right)=\zeta+\ln \operatorname{Im}[w]$ holds. Since $\mathscr{H}_{-\zeta}(\infty)$ is a horizontal line, $\rho\left(w, \mathscr{H}_{-\zeta}(\infty)\right)=\rho\left(w, \operatorname{Re}[w]+i e^{-\zeta}\right)$ holds. Apply 
(2.1) to the right-hand side of this equation together with $\operatorname{Im}[w]>e^{\zeta}>e^{-\zeta}$ by $e^{\zeta}>e^{1 / 2}>1$, we have the desired equality as

$$
\begin{aligned}
\rho\left(w, \mathscr{H}_{-\zeta}(\infty)\right) & =\rho\left(w, \operatorname{Re}[w]+i e^{-\zeta}\right) \\
& =\ln \frac{2 \operatorname{Im}[w]}{2 e^{-\zeta}} \\
& =\zeta+\ln \operatorname{Im}[w] .
\end{aligned}
$$

We finally show that for any $w \in \mathscr{S}_{\zeta}, \zeta+\ln \operatorname{Im}[w]>\rho\left(w, i e^{\zeta}\right)$ holds. By the formula (2.1), it is enough to show the following inequality:

$$
\sinh \frac{\zeta+\ln \operatorname{Im}[w]}{2}=\frac{e^{\zeta} \operatorname{Im}[w]-1}{2 \sqrt{e^{\zeta}} \sqrt{\operatorname{Im}[w]}}>\frac{\left|w-i e^{\zeta}\right|}{2 \sqrt{e^{\zeta}} \sqrt{\operatorname{Im}[w]}}=\sinh \frac{\rho\left(w, i e^{\zeta}\right)}{2},
$$

or equivalently $\left(e^{\zeta} \operatorname{Im}[w]-1\right)^{2}-\left|w-i e^{\zeta}\right|^{2}>0$, and it is shown as

$$
\begin{aligned}
& \left(e^{\zeta} \operatorname{Im}[w]-1\right)^{2}-\left|w-i e^{\zeta}\right|^{2} \\
& \quad=\left(e^{2 \zeta} \operatorname{Im}[w]^{2}-2 e^{\zeta} \operatorname{Im}[w]+1\right)-\left(\operatorname{Re}[w]^{2}+\operatorname{Im}[w]^{2}-2 e^{\zeta} \operatorname{Im}[w]+e^{2 \zeta}\right) \\
& \left.\quad \geq\left(e^{2 \zeta} \operatorname{Im}[w]^{2}-2 e^{\zeta} \operatorname{Im}[w]+1\right)-\left(\frac{1}{2}\right)^{2}+\operatorname{Im}[w]^{2}-2 e^{\zeta} \operatorname{Im}[w]+e^{2 \zeta}\right) \\
& \quad=\left(\left(e^{\zeta}\right)^{2}-1\right)\left(\operatorname{Im}[w]^{2}-1\right)-\left(\frac{1}{2}\right)^{2} \\
& \quad>\left(\left(e^{\frac{1}{2}}\right)^{2}-1\right)\left(\left(e^{\frac{1}{2}}\right)^{2}-1\right)-\left(\frac{1}{2}\right)^{2} \\
& \quad=\left(e-\frac{1}{2}\right)\left(e-\frac{3}{2}\right)>0,
\end{aligned}
$$

where the first inequality comes from $|\operatorname{Re}[w]|<1 / 2$ and the second inequality comes from $\operatorname{Im}[w]>e^{\zeta}>e^{1 / 2}$, since we assume that $w \in \mathscr{S}_{\zeta}$.

Since $R$ in Lemma 2.8 can always be chosen to satisfy $z \in \overline{\mathscr{D}_{R}(p)}$, we have the following corollary.

Corollary 2.9. Let $G$ be a Fuchsian group of finite coarea but not cocompact, and let $r \in \mathbb{R}$ be greater than both $R$ in Proposition 2.7 and $1 / 2$. For $z \in \mathbb{H}^{2}-\operatorname{Fix}(G)$, suppose that $z \notin D_{r}(z)$. Then there exists a parabolic fixed point $p$ of $G$ such that $z \in \mathscr{D}_{r}(p)$ and that $\rho\left(z, D_{r}(z)\right)=\rho\left(z, \mathscr{H}_{r}(p)\right)$.

\section{HAUSDORFF CONVERGENCE OF THE BOUNDARY OF TRUNCATED DiRICHLET POLYGONS}

Though the following fact, which is a generalization of Proposition 2.6 in [3], may be well-known, we will provide its proof.

Theorem 3.1. Let $G$ be a Fuchsian group of finite coarea but not cocompact, and let $r \in \mathbb{R}$ be greater than both $R$ in Proposition 2.7 and $1 / 2$. If a sequence of points $\left\{z_{n}\right\}_{n \in \mathbb{N}}$ in $\mathbb{H}^{2}-\operatorname{Fix}(G)$ has the limit $z \in \mathbb{H}^{2}-\operatorname{Fix}(G)$ with respect to the distance $\rho$, then a sequence of sets $\left\{\partial_{D} D_{r}\left(z_{n}\right)\right\}_{n \in \mathbb{N}}$ converges to a set $\partial_{D} D_{r}(z)$ in the Hausdorff topology. 
To prove this fact, we first prove the following lemma, which will be the key ingredient to prove not only Theorem 3.1 but also Lemma 4.3 .

Lemma 3.2. Let $G$ be a Fuchsian group of finite coarea but not cocompact, and let $r \in \mathbb{R}$ be greater than both $R$ in Proposition 2.7 and $1 / 2$. Let $\left\{z_{n}\right\}_{n \in \mathbb{N}}$ be a sequence of points in $\mathbb{H}^{2}-\operatorname{Fix}(G)$ with limit $z \in \mathbb{H}^{2}-\operatorname{Fix}(G)$ with respect to the distance $\rho$. Let $\left\{w_{n}\right\}_{n \in \mathbb{N}}$ be a sequence of points in $\mathbb{H}^{2}$ with $w_{n} \in \partial_{D} D_{r}\left(z_{n}\right)$ for any $n \in \mathbb{N}$. Let $W$ be the set of accumulation points of $\left\{w_{n}\right\}_{n \in \mathbb{N}}$. Then the following holds:

(1) The set $W$ is non-empty and contained in $\partial_{D} D_{r}(z)$.

(2) For any $w \in W$, there exist an element $g \in G$ and a subsequence $\left\{w_{n_{j}}\right\}_{j \in \mathbb{N}}$ converging to $w$ such that for any $j \in \mathbb{N}$, both $g \in S\left(z_{n_{j}}\right)$ and $w_{n_{j}} \in$ $\partial_{D} D_{r}\left(z_{n_{j}}\right) \cap L_{g}\left(z_{n_{j}}\right)$ hold.

Proof. We first see the non-emptiness of $W$, which is mentioned in (1) Finding a compact subset in $\mathbb{H}^{2}$ containing a subsequence of $\left\{w_{n}\right\}_{n \in \mathbb{N}}$ is enough to prove it.

We first consider the case that $z \in \overline{D_{r}(z)}$. Since $\left\{z_{n}\right\}_{n \in \mathbb{N}}$ is a converging sequence, there exists some $M^{\prime}>0$ such that $\left\{z_{n}\right\}_{n \in \mathbb{N}} \subset B_{M^{\prime}}(z)$. Set $M:=$ $2 M_{r}+M^{\prime}$. Then $\overline{B_{M}(z)}$ is the desired compact subset. Actually, we have $\overline{D_{r}\left(z_{n}\right)} \subset$ $\overline{B_{2 M_{r}}\left(z_{n}\right)}$ for any $n \in \mathbb{N}$, which implies

$$
w_{n} \in \overline{D_{r}\left(z_{n}\right)} \subset \overline{B_{2 M_{r}}\left(z_{n}\right)} \subset \overline{B_{M}(z)}
$$

for any $n \in \mathbb{N}$.

We next consider the case that $z \notin \overline{D_{r}(z)}$. We can apply Corollary 2.9 by our assumption of $r$. So there exists a parabolic fixed point $p$ of $G$ such that $z \in \mathscr{D}_{r}(p)$. The hyperbolic distance $\ell$ between $z$ and $D_{r}(z)$ coincides with the one between $z$ and $\mathscr{H}_{r}(p)$. Since $z_{n}$ converges to $z$, by dropping a finite number of terms if necessary, we may assume without loss of generality that for any $n \in \mathbb{N}, \rho\left(z, z_{n}\right)<\ell$ holds, which means that $\left\{z_{n}\right\}_{n \in \mathbb{N}} \subset \mathscr{D}_{r}(p)$. So, by Corollary 2.9 again, the hyperbolic distance $\ell_{n}$ between $z_{n}$ and $D_{r}\left(z_{n}\right)$ is positive, and it is provided by the distance from $z_{n}$ to $\mathscr{H}_{r}(p)$. So $\ell_{n} \leq 2 \ell$ for any sufficiently large $n$. Set $M:=2 M_{r}+3 \ell$ in this case. Then $\overline{B_{M}(z)}$ is the desired compact subset, since

$$
w_{n} \in \overline{D_{r}\left(z_{n}\right)} \subset \overline{B_{2 M_{r}+\ell_{n}}\left(z_{n}\right)} \subset \overline{B_{2 M_{r}+2 \ell}\left(z_{n}\right)} \subset \overline{B_{M}(z)}
$$

holds for any $n \in \mathbb{N}$.

Before proving the remaining part of (1), we prove (2), For a chosen $w \in W$, there exists a subsequence $\left\{w_{n_{i}}\right\}_{i \in \mathbb{N}}$ such that $w_{n_{i}}$ converges to $w$ as $i \rightarrow \infty$. Since $w_{n_{i}} \in \partial_{D} D_{r}\left(z_{n_{i}}\right)$, we can find a side-pairing transformation $f_{i} \in S\left(z_{n_{i}}\right)$ which sends $w_{n_{i}}$ to a point $f\left(w_{n_{i}}\right)$ in $\partial_{D} D_{r}\left(z_{n_{i}}\right)$ by Proposition 2.1. We thus have $\rho\left(w_{n_{i}}, f\left(w_{n_{i}}\right)\right) \leq M_{r}$. Combine (3.1) or (3.2) with this inequality and we have

$$
\rho\left(z, f_{i}(z)\right) \leq \rho\left(z, w_{n_{i}}\right)+\rho\left(w_{n_{i}}, f_{i}\left(w_{n_{i}}\right)\right)+\rho\left(f_{i}\left(w_{n_{i}}\right), f_{i}(z)\right) \leq 2 M+M_{r} .
$$

Since $G$ acts discontinuously on $\mathbb{H}^{2}$, there exist finitely many distinct $f_{i}$ satisfying (3.3) by Theorem 5.3.2 in [2]. Choose a subsequence $\left\{i_{j}\right\}_{j \in \mathbb{N}}$ of the indexes of $f_{i}$ such that $f_{i_{j}}$ are the same element $f$ in $G$. Set $n_{j}:=n_{i_{j}}$ and we have a subsequence $\left\{w_{n_{j}}\right\}_{j \in \mathbb{N}}$ such that for any $j \in \mathbb{N}, f=f_{n_{j}}$ and $w_{n_{j}} \in \partial_{D} D_{r}\left(z_{n_{j}}\right) \cap L_{f^{-1}}\left(z_{n_{j}}\right)$ hold. The desired $g$ is then chosen to be $f^{-1}$.

We finally prove the remaining part of (1) $W \subset \partial_{D} D_{r}(z)$. For a chosen $w \in W$, let $g \in S\left(z_{n_{j}}\right)$ and a subsequence $\left\{w_{n_{j}}\right\}_{j \in \mathbb{N}}$ of $\left\{w_{n}\right\}_{n \in \mathbb{N}}$ satisfying (2). We first see 
$w \in L_{g}(z)$. This is shown by the equations

$$
\begin{aligned}
\rho(z, w) & =\lim _{j \rightarrow \infty} \rho\left(z_{n_{j}}, w_{n_{j}}\right) \\
& =\lim _{j \rightarrow \infty} \rho\left(g\left(z_{n_{j}}\right), w_{n_{j}}\right) \\
& =\rho(g(z), w) .
\end{aligned}
$$

We next see that $w \in \partial D(z)$. As we have seen in (2), for any $j \in \mathbb{N}, w_{n_{j}} \in$ $\partial_{D} D_{r}\left(z_{n_{j}}\right) \cap L_{g}\left(z_{n_{j}}\right)$ holds. So $\rho\left(z_{n_{j}}, w_{n_{j}}\right) \leq \rho\left(f\left(z_{n_{j}}\right), w_{n_{j}}\right)$ holds for any $f \in G$. Take the limits of the both sides and we have $\rho(z, w) \leq \rho(f(z), w)$ for any $f \in G$, i.e., $w \in \overline{D(z)}$. This implies that $w \in \overline{D(z)} \cap L_{g}(z) \subset \partial D(z)$. We finally see that $w \in \partial_{D} D_{r}(z)$. Since $\pi\left(w_{n_{j}}\right) \in \pi\left(\overline{N_{r}}\right)$ and $\pi\left(\overline{N_{r}}\right)$ is compact, $\pi(w) \in \pi\left(\overline{N_{r}}\right)$ holds. Since $\overline{N_{r}} \cap \partial D(z)=\partial_{D} D_{r}(z)$, we have $w \in \partial_{D} D_{r}(z)$.

Proof of Theorem 3.1. By the definition of Hausdorff topology, all we need to prove is that, for any chosen $\varepsilon>0$, there exists some $N \in \mathbb{N}$ such that for any $n \geq N$, both of the following hold:

(a) $B_{\varepsilon}(w) \cap \partial_{D} D_{r}(z) \neq \emptyset$ for any $w \in \partial_{D} D_{r}\left(z_{n}\right)$, and

(b) $B_{\varepsilon}(w) \cap \partial_{D} D_{r}\left(z_{n}\right) \neq \emptyset$ for any $w \in \partial_{D} D_{r}(z)$.

We remark that Lemma 3.2 is used only in the proof of (a), but not in the proof of (b).

In order to prove (国) in the way of contradiction, we assume that there exists some $\varepsilon>0$ such that, for any $N \in \mathbb{N}$, there exist $n \geq N$ and $w_{n} \in \partial_{D} D_{r}\left(z_{n}\right)$ satisfying $B_{\varepsilon}\left(w_{n}\right) \cap \partial_{D} D_{r}(z)=\emptyset$. Lemma 3.2) provides an accumulation point $w$ of $\left\{w_{n}\right\}_{n \in \mathbb{N}}$ with $w \in \partial_{D} D_{r}(z)$. This is a contradiction for a large $n$ such that $\rho\left(w, w_{n}\right)<\varepsilon$.

In order to prove (b) in the way of contradiction, we assume that there exists some $\varepsilon>0$ such that, for any $N \in \mathbb{N}$, there exist $n \geq N$ and some $w \in \partial_{D} D_{r}(z)$ satisfying $B_{\varepsilon}(w) \cap \partial_{D} D_{r}\left(z_{n}\right)=\emptyset$. This assumption means that there exist a subsequence of $\left\{z_{n}\right\}_{n \in \mathbb{N}}$, again denoted by $\left\{z_{n}\right\}_{n \in \mathbb{N}}$ for simplicity, converging to $z$ as $n \rightarrow \infty$, and a point $w_{n} \in \partial_{D} D_{r}(z)$ for each $n \in \mathbb{N}$ such that

$$
B_{\varepsilon}\left(w_{n}\right) \cap \partial_{D} D_{r}\left(z_{n}\right)=\emptyset .
$$

Since the set $\overline{\partial_{D} D_{r}(z)}$ is compact, by passing to a subsequence if necessary, we can assume that the sequence $\left\{w_{n}\right\}_{n \in \mathbb{N}}$ converges to a point $w_{0}$ in $\overline{\partial_{D} D_{r}(z)}$. In particular, we can assume also that $\rho\left(w_{0}, w_{n}\right)<\varepsilon / 2$ holds for any $n \in \mathbb{N}$. Then $\partial_{D} D_{r}\left(z_{n}\right) \subset \mathbb{H}^{2}-B_{\varepsilon / 2}\left(w_{0}\right)$ holds as

$$
\rho\left(u, w_{0}\right) \geq \rho\left(u, w_{n}\right)-\rho\left(w_{n}, w_{0}\right)>\varepsilon-\frac{\varepsilon}{2}=\frac{\varepsilon}{2}
$$

for any $u \in \partial_{D} D_{r}\left(z_{n}\right)$. Since $D_{r}(z)$ is a polygon with finitely many sides, there exists a point $w^{\prime}$ of a side of $D_{r}(z)$ with $\rho\left(w_{0}, w^{\prime}\right)<\varepsilon / 4$. We replace $\varepsilon$ by a number less than $\varepsilon / 4$ so that $B_{\varepsilon}\left(w^{\prime}\right)$ is included in the truncated Nielsen region $N_{r}$ and that $B_{\varepsilon}\left(w^{\prime}\right) \cap \partial_{D} D_{r}\left(z_{n}\right)=\emptyset$ for any $n \in \mathbb{N}$. This condition also implies that $B_{\varepsilon}\left(w^{\prime}\right)$ is disjoint from $\partial D\left(z_{n}\right)$ for any $n \in \mathbb{N}$. Passing to a subsequence if necessary, we assume that either one of the following holds:

(b)i) $B_{\varepsilon}\left(w^{\prime}\right) \subset D\left(z_{n}\right)$ for any $n \in \mathbb{N}$, or

(b)ii) $B_{\varepsilon}\left(w^{\prime}\right) \subset \mathbb{H}^{2}-\overline{D\left(z_{n}\right)}$ for any $n \in \mathbb{N}$. 
If (b,i) occurs, then $B_{\varepsilon}\left(w^{\prime}\right)$ is fully contained in $\overline{D(z)}$, since we have $\rho(u, z) \leq$ $\rho(u, g(z))$ for any $u \in B_{\varepsilon}\left(w^{\prime}\right)$ and any $g \in G$, by taking the limit of $\rho\left(u, z_{n}\right) \leq$ $\rho\left(u, g\left(z_{n}\right)\right)$. This contradicts the assumption that $w^{\prime} \in \partial_{D} D_{r}(z) \subset \partial D(z)$. If (b.ii) occurs, then, for any $u \in B_{\varepsilon}\left(w^{\prime}\right)$ and any $n \in \mathbb{N}$, there exists some element $g_{n} \in G-\{$ id $\}$ such that $\rho\left(u, z_{n}\right)>\rho\left(u, g_{n}\left(z_{n}\right)\right)$. Then $\rho\left(u, g_{n}(z)\right)$ is bounded as

$$
\begin{aligned}
\rho\left(u, g_{n}(z)\right) & \leq \rho\left(u, g_{n}\left(z_{n}\right)\right)+\rho\left(g_{n}\left(z_{n}\right), g_{n}(z)\right) \\
& <\rho\left(u, z_{n}\right)+\rho\left(z_{n}, z\right) \\
& \leq \rho(u, z)+2 \rho\left(z, z_{n}\right),
\end{aligned}
$$

and $z_{n}$ converges to $z$. So, there exists an element $g \in G-\{$ id $\}$ such that $g=g_{n}$ for infinitely many $n \in \mathbb{N}$. By taking the limit in $\rho\left(u, z_{n}\right)>\rho\left(u, g\left(z_{n}\right)\right)$, we have $\rho(u, z) \geq \rho(u, g(z))$. Thus $u \in \mathbb{H}^{2}-D(z)$. Since $u$ is an arbitrary point of $B_{\varepsilon}\left(w^{\prime}\right)$, we conclude that $B_{\varepsilon}\left(w^{\prime}\right) \subset \mathbb{H}^{2}-D(z)$. This also contradicts the assumption that $w^{\prime} \in \partial_{D} D_{r}(z)$.

\section{OpenNess OF The SET OF REgular POINTS}

Let $\operatorname{PS}(z)$ be the set of points having the same side-paring transformations of $D(z)$ :

$$
\operatorname{PS}(z):=\left\{w \in \mathbb{H}^{2} \mid S(w)=S(z)\right\} .
$$

The main result in this section is the following theorem, which is a generalization of Proposition 3.3 in [3] and will be used to prove Theorem [5.1

Theorem 4.1. For a Fuchsian group $G$ of finite coarea and for a regular point $z \in \operatorname{Reg}(G)$, the set $\operatorname{PS}(z)$ is open in $\mathbb{H}^{2}$.

An immediate corollary, though it will not be used to prove Theorem 5.1, is the openness of $\operatorname{Reg}(G)$, since it is the union of $\operatorname{PS}(z)$ for any $z \in \operatorname{Reg}(G)$. Similar results have already been obtained as Corollary 2.1 in [5] and Theorem 3.4 in [3].

Corollary 4.2. For a Fuchsian group $G$ of finite coarea, the set $\operatorname{Reg}(G)$ of regular points is open in $\mathbb{H}^{2}$.

Our proof of Theorem 4.1 is based on a series of lemmas, which are prepared from now on.

Lemma 4.3. For a Fuchsian group $G$ of finite coarea, any sequence of points $\left\{z_{n}\right\}_{n \in \mathbb{N}}$ in $\mathbb{H}^{2}-\operatorname{Fix}(G)$ with limit $z \in \mathbb{H}^{2}-\operatorname{Fix}(G)$ has a subsequence $\left\{z_{n_{j}}\right\}_{j \in \mathbb{N}}$ such that $S(z) \subset \bigcap_{j \in \mathbb{N}} S\left(z_{n_{j}}\right)$.

Proof. It is enough to show that, for each $f \in S(z)$, there exists a subsequence $\left\{z_{n_{j}}\right\}_{j \in \mathbb{N}}$ with $f \in \bigcap_{j \in \mathbb{N}} S\left(z_{n_{j}}\right)$. Then choose any $f_{1}$ from $S(z)$ and a subsequence $\left\{z_{n_{j}}\right\}_{j \in \mathbb{N}}$ such that $f_{1} \in \bigcap_{j \in \mathbb{N}} S\left(z_{n_{j}}\right)$. Next choose a second element $f_{2}$ from $S(z)$ and extract a subsequence from $\left\{z_{n_{j}}\right\}_{j \in \mathbb{N}}$ so that $f_{2} \in \bigcap_{j \in \mathbb{N}} S\left(z_{n_{j}}\right)$. Since $S(z)$ is a finite set, by repeating this procedure we obtain a desired subsequence.

Recall that $E(z)$ is the set of vertices of $D(z)$ that lie on $\partial_{\infty} \mathbb{H}^{2}$. By Lemma 2.5, every point in $E(z)$ is a parabolic fixed point of $G$. Since $D(z)$ has finitely many sides, we can choose a large number $r$ such that the following properties hold for all vertices $p \in E(z)$ :

- $r$ satisfies the condition in Theorem 3.1, i.e., $r$ is greater than both $R$ in Proposition 2.7 and 1/2; 
- $\partial D(z)$ meets $\mathscr{D}_{r}(p)$ in two sides both ending at $p$;

- $\mathscr{H}_{r}(p)=\partial \mathscr{D}_{r}(p)$ cuts the above two sides in their interior points.

We remark that the two sides above are not necessarily paired by an element of $S(z)$, since we do not assume here that $z$ is a regular point. Let $f \in S(z)$ be a side-pairing transformation. There exists a side $s \subset \partial D(z)$ such that $s=\overline{D(z)} \cap f(\overline{D(z)}) \subset$ $L_{f}(z)$. Let $w$ be a point in the intersection of $\partial_{D} D_{r}(z)$ and the interior of $s$ with respect to the relative topology of $s$. For this $w$, (b) in the proof of Theorem 3.1 provides a point $w_{n} \in \partial_{D} D_{r}\left(z_{n}\right)$ for each $n \in \mathbb{N}$ such that $w_{n}$ converges to $w$ as $n \rightarrow \infty$ in $\mathbb{H}^{2}$. Apply Lemma 3.2(2) to the sequence $\left\{w_{n}\right\}_{n \in \mathbb{N}}$ and we can find a subsequence $\left\{w_{n_{j}}\right\}_{j \in \mathbb{N}}$ and $h \in \bigcap_{j \in \mathbb{N}} S\left(z_{n_{j}}\right)$ such that $w_{n_{j}} \in L_{h}\left(z_{n_{j}}\right)$.

In order to see that the subsequence $\left\{z_{n_{j}}\right\}_{j \in \mathbb{N}}$ is the desired one, it is enough to show that $h=f$. Since $w_{n_{j}} \in L_{h}\left(z_{n_{j}}\right)$ together with the continuity of the bisector, we have $w \in L_{h}(z)$. Since $w \in s$, we also have $w \in L_{f}(z)$. So we have $w \in L_{f}(z) \cap L_{h}(z)$. Since $w$ is taken from the interior of $s$, it cannot be a vertex of $D(z)$. So, by the uniqueness of the side-paring transformation, we have $L_{f}(z)=L_{h}(z)$, which implies $f=h$.

Lemma 4.4. For a Fuchsian group $G$ of finite coarea and a point $z \in \mathbb{H}^{2}-\operatorname{Fix}(G)$, there exists an open neighborhood $U \subset \mathbb{H}^{2}-\operatorname{Fix}(G)$ of $z$ such that for any $u \in U$, $S(z) \subset S(u)$ holds.

Proof. We first show that for any $f \in S(z)$, there exists an open neighborhood $U_{f} \subset \mathbb{H}^{2}-\operatorname{Fix}(G)$ of $z$ such that for any $u \in U_{f}$, the map $f \in S(u)$ holds. By way of contradiction, assume that there exist some $z \in \mathbb{H}^{2}-\operatorname{Fix}(G)$ and some $f \in S(z)$ such that for any open neighborhood $U \subset \mathbb{H}^{2}-\operatorname{Fix}(G)$ of $z$, there exists some $u \in U$ with $f \notin S(u)$. Then there exists a positive number $n_{0}$ such that $B_{1 / n_{0}}(z) \cap \operatorname{Fix}(G)=\emptyset$ as $\operatorname{Fix}(G)$ is a discrete set of points. Regarding $U$ above as $B_{1 / n}(z)$ we can find a point $z_{n} \in B_{1 / n}(z)$ for any $n>n_{0}$ such that $f \notin \bigcap_{n>n_{0}} S\left(z_{n}\right)$, which contradicts Lemma 4.3 .

So there exists an open neighborhood $U_{f} \subset \mathbb{H}^{2}-\operatorname{Fix}(G)$ of $z$ such that for any $u \in U_{f}, f \in S(u)$ holds. The set $U:=\bigcap_{f \in S(z)} U_{f}$ is a desired neighborhood of $z$ as $S(z)$ is a finite set.

Proof of Theorem 4.1. For any $w \in \operatorname{PS}(z)$, let $U_{w}$ be an open neighborhood of $w$ obtained in Lemma 4.4. We show that $U_{w} \subset \operatorname{PS}(z)$, i.e., $S(u)=S(z)$ for any $u \in U_{w}$. For any such $u$, the inclusion $S(z)=S(w) \subset S(u)$ comes from the definition of $U_{w}$. We thus have $|S(z)| \leq|S(u)|$. Since $z \in \operatorname{Reg}(G), S_{G}=|S(z)|$ is the maximal number of sides. So we have $|S(z)|=|S(u)|$. We can conclude that $S(u)=S(z)$ as $S(z)$ is a finite set.

\section{EXIstence OF EXCEPTIONAL POINTS}

It is unknown whether the $\operatorname{set} \operatorname{Exc}(G)$ of all exceptional points is empty or not at this point. For cocompact Fuchsian groups $G$, Theorem 4.3 in [3] shows that $\operatorname{Exc}(G)$ contains uncountably many points. We provide a generalization of this result for Fuchsian groups of finite coarea.

Theorem 5.1. For any Fuchsian group $G$ of finite coarea, the set $\operatorname{Exc}(G)$ of exceptional points contains uncountably many points. 
We remark that the proof of Theorem 5.1 is not a straightforward generalization of Fera's one.

Example 5.2. Let $\triangle$ be the open triangular region bounded by three geodesics

$$
\begin{aligned}
& m_{1}:=\left\{z \in \mathbb{H}^{2}|| z \mid=1\right\}, \\
& m_{2}:=\left\{z \in \mathbb{H}^{2} \mid \operatorname{Re}[z]=1\right\}, \\
& m_{3}:=\left\{z \in \mathbb{H}^{2} \mid \operatorname{Re}[z]=-1\right\} .
\end{aligned}
$$

Let $R_{j} \in \mathrm{SL}(2, \mathbb{R})$ be the reflection in $m_{j}$ for $j=1,2,3$. We define

$$
\begin{aligned}
& T_{1}:=R_{1} \circ R_{3}=\left(\begin{array}{cc}
0 & -1 \\
1 & 2
\end{array}\right), \\
& T_{2}:=R_{2} \circ R_{1}=\left(\begin{array}{cc}
2 & -1 \\
1 & 0
\end{array}\right), \\
& T_{3}:=T_{2} \circ T_{1}=\left(\begin{array}{ll}
1 & 4 \\
0 & 1
\end{array}\right),
\end{aligned}
$$

and consider the Fuchsian group generated by $T_{1}$ and $T_{2}$. For any $z \in \triangle, D(z)$ is the open hexagonal region bounded by the bisection lines $L_{T_{1}^{ \pm 1}}(z), L_{T_{2}^{ \pm 1}}(z)$, $L_{T_{3}^{ \pm 1}}(z)$ by Theorem 24 in [8]. So $S(z)=\left\{T_{1}^{ \pm 1}, T_{2}^{ \pm 1}, T_{3}^{ \pm 1}\right\}$. On the other hand, again by Theorem 24 in [], $D(i)$ is the open quadratic region $\triangle \cup m_{1} \cup R_{1}(\triangle)$ and $S(i)=\left\{T_{1}^{ \pm 1}, T_{2}^{ \pm 1}\right\}$. So $T_{3}$ belongs to $S(z)$ for any $z \in \triangle$ but not to $S(i)$. The two sides of $D(z)$ which are paired by $T_{3}$ leave any compact subset of $\mathbb{H}^{2}$ as $z$ approaches $i$ from the inside of $\triangle$. This fact makes the situation difficult when we try to generalize Theorem 4.3 in [3] to Fuchsian groups of finite coarea but not cocompact.

From now on, we regard $\mathbb{H}^{2}$ as the so-called Poincaré disk model:

$$
\mathbb{H}^{2}:=\{z \in \mathbb{C}|| z \mid<1\} .
$$

Then $\partial_{\infty} \mathbb{H}^{2}$ is the unit circle centered at the origin 0 . A point $\xi \in \partial_{\infty} \mathbb{H}^{2}$ is called a transitive limit point (or a Myrberg point) of $G$ if the projection to the unit tangent bundle of the quotient surface $\mathbb{H}^{2} / G$ of the unit tangent field along a geodesic ray from any point in $\mathbb{H}^{2}$ to $\xi$ is dense (and hence the projections to $\mathbb{H}^{2} / G$ of all geodesic rays ending at $\xi$ is dense). See $\S 2.2$ in 6 . Let $T_{G}$ be the set of all transitive limit points of $G$. If $G$ is of finite coarea (or if, more generally, $G$ is of divergence-type), it is shown in [7] that the measure of $T_{G}$ is $2 \pi$.

Proof of Theorem 5.1. By replacing $G$ by its suitable conjugate if necessary, we can assume without loss of generality that 0 is a regular point for $G$. We delete the radial projection of $\operatorname{Fix}(G)$ from $T_{G}$, and we denote the remaining set again by $T_{G}$. Our new $T_{G}$ still has measure $2 \pi$ as $\operatorname{Fix}(G)$ is a countable set. It is thus enough to construct an injection from $T_{G}$ to $\operatorname{Exc}(G)$ to complete the proof.

For any $\xi \in T_{G}$, let $\gamma_{\xi}:=\left\{t \xi \in \mathbb{H}^{2} \mid 0<t<1\right\}$ be a geodesic from 0 to $\xi$. We will see that for any $\xi \in T_{G}, \gamma_{\xi} \cap \operatorname{Exc}(G) \neq \emptyset$ holds. The desired injection is then given by corresponding $\xi \in T_{G}$ to a point in $\gamma_{\xi} \cap \operatorname{Exc}(G)$.

Let $U \subset \operatorname{PS}(0) \cap D(0)$ be an open neighborhood of 0 . The image $\pi\left(\gamma_{\xi}\right)$ of $\gamma_{\xi}$ on the quotient surface $\mathbb{H}^{2} / G$ visits $\pi(U)$ infinitely many times, since $\xi \in \partial_{\infty} \mathbb{H}^{2}$ is a transitive limit point. So there exist a sequence $\xi_{n}:=t_{n} \xi$ on $\gamma_{\xi}$ with $t_{n}$ converging to 1 as $n \rightarrow \infty$ and a sequence of distinct elements $h_{n} \in G$ such 
that $\xi_{n} \in h_{n}(U)$ holds. We then have $S\left(\xi_{n}\right)=S\left(h_{n}(0)\right)=h_{n} S(0) h_{n}^{-1}$, since $h_{n}(U) \subset h_{n}(\mathrm{PS}(0) \cap D(0)) \subset h_{n}(\mathrm{PS}(0)) \cap h_{n}(D(0))$.

The subset $\gamma_{\xi} \cap \operatorname{PS}(0)$ of $\gamma_{\xi}$ is non-empty and open with respect to the relative topology, since $0 \in \operatorname{Reg}(G)$ and $\operatorname{PS}(0)$ is open in $\mathbb{H}^{2}$ by Theorem 4.1. Furthermore, $\gamma_{\xi}$ is a proper subset, that is, $\gamma_{\xi} \not \subset \operatorname{PS}(0)$. Actually, if $\gamma_{\xi} \subset \operatorname{PS}(0)$, then $\xi_{n} \in \operatorname{PS}(0)$ for any $n \in \mathbb{N}$, which means that $S\left(\xi_{n}\right)=S(0)$. So, for any $n \in \mathbb{N}, h_{n} S(0) h_{n}^{-1}=$ $S(0)$ holds. Since $S(0)$ is a finite set, there exists a permutation $\sigma$ of $S(0)$ such that for infinitely many $n \in \mathbb{N}$ and for any $f \in S(0), h_{n} f h_{n}^{-1}=\sigma(f)$ holds. In particular, there exist distinct $m, n \in \mathbb{N}$ such that for any $f \in S(0), h_{n} f h_{n}^{-1}=h_{m} f h_{m}^{-1}$ holds. So $h_{m}^{-1} h_{n}$ commutes with all elements of $G$ as $S(0)$ generates $G$. The element $h_{m}^{-1} h_{n}$ must be the trivial element id as $G$ is non-elementary. This contradicts the assumption that $h_{n} \neq h_{m}$.

We now have a non-empty proper open subset $\gamma_{\xi} \cap \mathrm{PS}(0)$ of $\gamma_{\xi}$ with respect to the relative topology. If $\gamma_{\xi} \subset \operatorname{Reg}(G)$, then the geodesic segment $\gamma_{\xi}$ must be covered by more than one disjoint open sets of the form $\operatorname{PS}(w)$. However, this contradicts the connectivity of $\gamma_{\xi}$. So we have $\gamma_{\xi} \not \subset \operatorname{Reg}(G)$, which implies $\gamma_{\xi} \cap \operatorname{Exc}(G) \neq \emptyset$, since $\gamma_{\xi} \subset \operatorname{Reg}(G) \cup \operatorname{Exc}(G)$.

\section{ACKNOWLEDGMENTS}

The authors wishes to express their sincere gratitude to the referees for their helpful suggestions. In particular, they informed us about Fera and Lazowski's result 4], in which they had already reached the same conclusion as ours by a different method.

\section{REFERENCES}

[1] A. F. Beardon, The geometry of discrete groups, Discrete groups and automorphic functions (Proc. Conf., Cambridge, 1975), Academic Press, London, 1977, pp. 47-72. MR0474012

[2] Alan F. Beardon, The geometry of discrete groups, Graduate Texts in Mathematics, vol. 91, Springer-Verlag, New York, 1983. MR698777

[3] Joseph Fera, Exceptional points for cocompact Fuchsian groups, Ann. Acad. Sci. Fenn. Math. 39 (2014), no. 1, 463-472, DOI 10.5186/aasfm.2014.3917. MR3186824

[4] Joseph Fera and Andrew Lazowski, Exceptional points for finitely generated Fuchsian groups of the first kind, Advances in Geometry, (published online ahead of print), 2019.

[5] Marjatta Näätänen, On the stability of identification patterns for Dirichlet regions, Ann. Acad. Sci. Fenn. Ser. A I Math. 10 (1985), 411-417, DOI 10.5186/aasfm.1985.1045. MR802503

[6] Peter J. Nicholls, The ergodic theory of discrete groups, London Mathematical Society Lecture Note Series, vol. 143, Cambridge University Press, Cambridge, 1989. MR.1041575

[7] Saburo Shimada, On P. J. Myrberg's approximation theorem on Fuchsian groups, Mem. Coll. Sci. Univ. Kyoto Ser. A. Math. 33 (1960/61), 231-241, DOI 10.1215/kjm/1250775909. MR 158074

[8] Yuriko Umemoto, On Dirichlet fundamental domains for Fuchsian groups, Master Thesis, Graduate School of Science, Osaka City University, 2011.

Department of Mathematics, Shimane University, Matue 690-8504, Japan

Email address: tosihiro@riko.shimane-u.ac.jp

Faculty of Mathematics and Physics, Institute of Science and Engineering, Kanazawa UNIVERSITY, ISHIKAWA 920-1192, JAPAN

Email address: ushijima@se.kanazawa-u.ac.jp 